\title{
Efficacy of random primer-pair arrays in plant genome analysis: a case study of Cucumis (Cucurbitaceae) for identification of wild and cultivated species
}

\author{
E.M. Gatphoh, S.K. Sharma, K. Rajkumari and S. Rama Rao \\ Plant Biotechnology Laboratory, Department of Biotechnology and Bioinformatics, \\ North-Eastern Hill University, Shillong, Meghalaya, India \\ Corresponding author: S. Rama Rao \\ E-mail: srrao22@yahoo.com / srrao@mail.com
}

Genet. Mol. Res. 10 (3): 1416-1426 (2011)

Received September 28, 2010

Accepted January 4, 2011

Published July 19, 2011

DOI 10.4238/vol10-3gmr1083

\begin{abstract}
The efficacy of random primer-pair arrays compared to conventional RAPD method with a single decamer primer was evaluated using DNA from two species of Cucumis. The banding patterns of amplicons revealed enhanced utility of primer-pair arrays over conventional RAPDs, producing more bands and a higher degree of polymorphism, both at intraand inter-specific levels. Amplification produced by both methods clearly distinguished a wild from a cultivated species of the genus Cucumis. The main advantage of the primer-pair RAPD over single-primer-based RAPD is the increase in the number of reactions and amplification products in the form of novel/unique bands with a limited number of primers. It also enables the generation of reliable amplicons with a large number of polymorphic bands, which can be linked to gene-governing traits, allowing sequence-characterized partial genome analysis.
\end{abstract}

Key words: Primer-pair arrays; RAPD; Novel bands; Efficacy; Genetic variation 


\section{INTRODUCTION}

Plant molecular biological tools for the detection of genetic variation by using polymerase chain reaction (PCR) rely on sequence information to design target-specific primers (Innis et al., 1990). Several novel types of marker systems that use PCR without prior information on target DNA sequences have been described. However, these systems make use of universal sets of short oligonucleotides that amplify the DNA fragments randomly even with single-primers under low-stringency conditions. This strategy has enabled DNA polymorphisms to be detected by single-primer amplification (Sharma et al., 2010) such as random amplified polymorphic DNA (RAPD) analysis (Williams et al., 1990), arbitrarily primed PCR analysis (Welsh and McClelland, 1990) and DNA amplification fingerprinting analysis (Caetano-Anolles et al., 1991). RAPD markers offer quick screening of different regions of the genome for genetic polymorphism. The conventional RAPD procedure uses a single-decamer primer to amplify short stretches of the genome by PCR. The RAPD marker system in particular has found widespread application in plant molecular biology, having been used for the detection of genetic variation, construction of linkage maps in various plants and animals (Hunt and Page Jr., 1995; Cushwa and Medrano, 1996; Huang et al., 2003), and in bulk segregant analysis for identifying markers linked to genes of interest (Williams et al., 1993). For example, the Arabidopsis thaliana's omega-3 desaturase gene $\mathrm{fad} 3$ and the disease-resistant gene RPS2 and various other genetic traits have been cloned by this approach (Arondel et al., 1992; Bent et al., 1994; Hu et al., 1995). A prerequisite for map-based cloning is the development of a highly saturated map in the region containing the gene of interest and used as starting points for chromosomal walking and eventually finding and cloning the target gene (Hu et al., 1995).

The present investigation basically deals with the amplification of different random primers with a few collections of two Cucumis species and further amplification of paired arrays of two best amplified primers individually as well as in pairs to test the reproducibility and the potential to amplify the long paired sequences in the genome. Interestingly, new bands were amplified in various primer-pair arrays. They had homology in the bands of larger molecular weight DNA fragments and at the same time resulted in certain novel bands representing lower molecular weight fragments that were revealed on the gel. Based on such preliminary observations, the authors propose to make a case for the efficacy of various primer-pair array-based RAPD amplification reactions and their relevance to understand the approach to develop the species-specific sequence-characterized amplified regions (SCAR) to map a second marker, which can be linked to various gene-governing traits.

\section{MATERIAL AND METHODS}

\section{Plant material}

Four samples of cultivated species of Cucumis, i.e., C. sativus, with collection numbers PBL-CS-01, PBL-CS-02, PBL-CS-03, PBL-CS-04, and one accession of C. hardwickii (wild), with collection number PBL-CH-01, were collected from the local habitat (Table 1). 


\begin{tabular}{|c|c|c|}
\hline Sample No. & Species name & Collection No. \\
\hline 1 & Cucumis sativus & PBL-CS-01 \\
\hline 2 & Cucumis sativus & PBL-CS-02 \\
\hline 3 & Cucumis sativus & PBL-CS-03 \\
\hline 4 & Cucumis sativus & PBL-CS-04 \\
\hline 5 & Cucumis hardwickii & PBL-CH-01 \\
\hline
\end{tabular}

\section{DNA extraction, PCR amplification and data analysis}

The frozen leaves were ground and powdered in a pre-chilled mortar using liquid nitrogen, and the DNA was then extracted by the method described by Murray and Thomson (1980). The DNA extracted from the plant material, purified for protein fraction and treated with RNase-A was re-precipitated using pre-chilled absolute ethanol and subsequently dissolved in Tris-EDTA (TE) buffer. The quality of DNA was checked by mupid gel electrophoresis with $0.85 \%(\mathrm{w} / \mathrm{v})$ agarose in $1 \mathrm{X}$ TAE.

Varying concentrations of i) template DNA (20, 30, 40, 50 and $60 \mathrm{ng})$; ii) Taq DNA polymerase (0.5-2 U), and iii) $\mathrm{Mg}^{2+}$ salt $(1-5 \mathrm{mM})$ were used to optimize the reaction conditions of the PCR using one specific accession. As many as 36 RAPD primers from the kits OPA, OPB, OPC, OPD, OPAA, and OPBA (Operon Technologies, USA) were assayed with selected collections to identify primers that were reproducible and generate the most polymorphic patterns. As mentioned earlier, of five different concentrations, $50 \mathrm{ng}$ template DNA was found to be ideal as it yielded the maximum number of reproducible bands. Similarly, $1.5 \mathrm{mM}$ $\mathrm{MgCl}_{2}$ and $1 \mathrm{U}$ Taq DNA polymerase have yielded ideal results among the various concentrations tested. Twelve RAPD decamer primers (Table 2) were selected for the final amplification program as others produced sub-optimal, indistinct, or monomorphic amplification products during the analysis of five collections of two Cucumis species. For primer-pairs, of 69 possible combinations, 26 combinations were used for the final amplification as the others were not reproducible and yielded unclear banding patterns. Once these conditions were established, amplification reactions including both single-primer amplification reaction and two-primer array for all the genotype primers were thereafter carried out as per these optimized conditions.

\begin{tabular}{|c|c|c|c|c|c|}
\hline Primer name & Primer sequence $\left(5^{\prime}-3^{\prime}\right)$, length & $\begin{array}{l}\text { Total No. } \\
\text { of bands }\end{array}$ & $\begin{array}{c}\text { No. of polymorphic } \\
\text { bands }\end{array}$ & $\%$ of polymorphism & $\begin{array}{l}\text { Range of molecular } \\
\text { weight }(\mathrm{kb})\end{array}$ \\
\hline OPD-8 & GTGTGCCCCA (10 mer) & 7 & 3 & 42.85 & $0.50-1.5$ \\
\hline OPBA-14 & TCGGGAGTGG (10 mer) & 4 & 0 & 0.0 & $0.45-1.8$ \\
\hline OPBA-13 & AGGGCGAATG (10 mer) & 8 & 5 & 62.5 & $0.40-1.3$ \\
\hline OPAA-1 & AGACGGCTCC (10 mer) & 6 & 1 & 16.66 & $0.50-1.4$ \\
\hline OPAA-2 & GAGACCAGAC (10 mer) & 7 & 2 & 28.57 & $0.45-1.5$ \\
\hline OPAA-4 & AGGACTGCTC (10 mer) & 6 & 2 & 33.33 & $0.45-1.8$ \\
\hline OPBA-16 & CCACGCATCA (10 mer) & 6 & 5 & 83.33 & $0.40-1.8$ \\
\hline OPBA-19 & CCATCCGTTG (10 mer) & 7 & 2 & 28.57 & $0.45-1.8$ \\
\hline OPAA-11 & ACCCGACCTG (10 mer) & 9 & 6 & 66.66 & $0.45-2.3$ \\
\hline OPAA-14 & AACGGGCCAA (10 mer) & 10 & 7 & 70.00 & $0.40-2.0$ \\
\hline OPAA-12 & GGACCTCTTG (10 mer) & 9 & 6 & 66.66 & $0.45-2.0$ \\
\hline OPC-11 & AAAGCTGCGG (10 mer) & 7 & 2 & 28.57 & $0.45-2.3$ \\
\hline Total number & & 75 & 41 & 54.66 & $0.40-2.3$ \\
\hline
\end{tabular}


On the basis of PCR optimization and primer survey, all further reactions were performed with $50 \mathrm{ng}$ template DNA, 30 pmol decamer primer (both in single- and two-primer array), $200 \mu \mathrm{M}$ each dNTP, $1.5 \mathrm{mM} \mathrm{Mg}^{2+}$, and $1 \mathrm{U}$ Taq DNA polymerase (Bangalore Genei, India) making up a final volume of $25 \mu \mathrm{L}$. The primer-pair was designed in such a way that each pair comprised two primers (in pmol) in the ratios of $1: 2,1: 1$ and $2: 1$, respectively, to optimize their concentration. The reaction components were maintained at these concentrations in all further PCRs. Reactions were set for PCR cycle at $94^{\circ} \mathrm{C}$ for $3 \mathrm{~min}$ followed by 40 cycles at $94^{\circ} \mathrm{C}$ for $1 \mathrm{~min}, 35^{\circ} \mathrm{C}$ for $1 \mathrm{~min}$, and $72^{\circ} \mathrm{C}$ for $1 \mathrm{~min}$ and $30 \mathrm{~s}$, with a final extension at $72^{\circ} \mathrm{C}$ for $7 \mathrm{~min}$ in a Multigene thermal cycler (Labnet International, Inc., USA). After completion of the amplification, 2.5 $\mu \mathrm{L} 10 \mathrm{X}$ bromophenol blue dye was added to the samples, and the amplified DNA was electrophoresed on $1.5 \%(\mathrm{w} / \mathrm{v})$ agarose gel in $1 \mathrm{X}$ TAE buffer at $65-70 \mathrm{~V}$ for 3-4 h. PCR and electrophoretic conditions remained the same for both the single-random primer and two-random primer array amplification reactions. Agarose gel photographs were taken using a gel documentation system (Gel Logic 100 Imaging System, Kodak) and band scoring for presence $(+)$ or absence (-) of the amplified bands and their sizes were determined with reference to known 500-bp DNA markers.

A data set of band profiles was scored manually from the gel profiles and included only the well-separated and distinct bands. The data for amplicons were scored cumulatively, first for all the primers as in a conventional RAPD technique and secondly for primer-pair array individually. Subsequently, the data sets from first and second approaches were combined together for the final neighbor-joining analysis. A pair-wise matrix of distances between genotypes was determined cumulatively for all three methods by Jaccard coefficient (Jaccard, 1901) using the NTSYS-pc 2.02k program. This distance matrix was used to compute a single consensus tree using the same program.

\section{RESULTS}

\section{Analysis of conventional RAPD versus random primer-pair array amplification reactions and comparison of clustering pattern}

While performing a single-random primer amplification reaction with all five DNA samples of two Cucumis species, a total of 75 amplification products were scored (Figure 1 and Table 2). The average number of amplification products formed was 6.25 with a maximum of 10 with OPAA-14 and a minimum of 4 with OPBA-14. The size of the amplification products varied for each primer and the range was $0.40-2.3 \mathrm{~kb}$. The amplification products using 12 primers were distinguished as monomorphic and polymorphic bands. The highest percentage of polymorphic bands observed was $83.33 \%$ with an average of $54.66 \%$ polymorphism. On the other hand, various pair arrays of the random primers amplified yielded a total of $164 \mathrm{am}-$ plification products scored, which exhibited an overall polymorphism of 56.09\% (Tables 3 and 4). The average number of amplification products formed was 6.30 (Table 4) with a maximum of 10 with a combination of OPBA-16 and OPC-11 and a minimum of 2 with a combination of OPAA-14 and OPD-8. The size of the amplification products varied for each primer and the range was $0.3-2.7 \mathrm{~kb}$. Pooled data of both techniques revealed an average polymorphism of $55.64 \%$ (Table 4 ). 


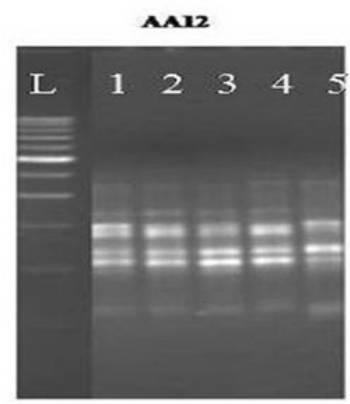

AnI

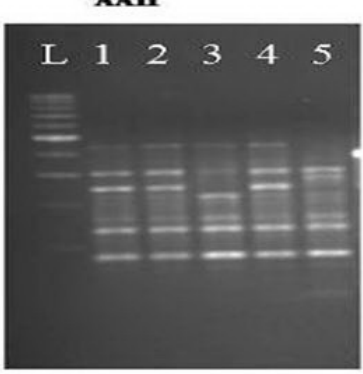

CII

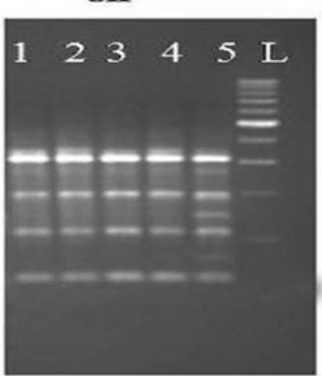

BA16
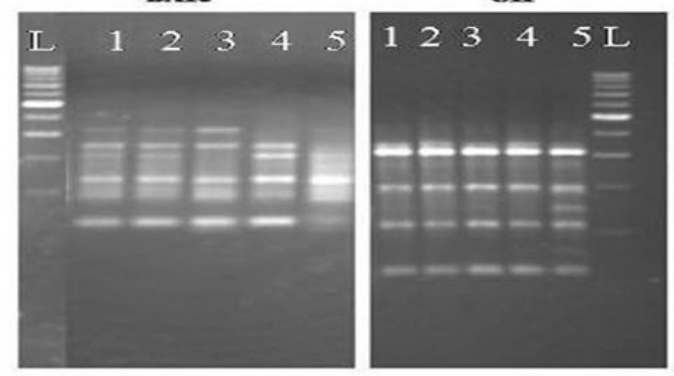

AN12 + AN2

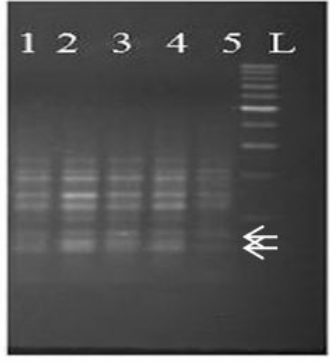

ANII + BAIS

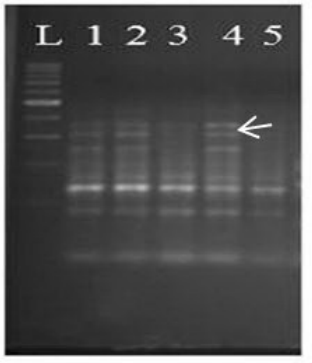

CW + EAIS

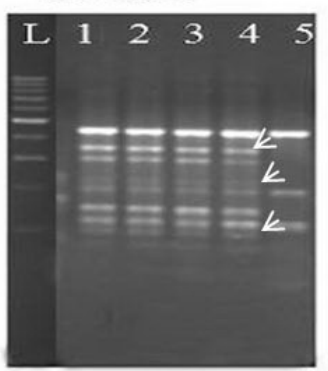

BA16 + CII

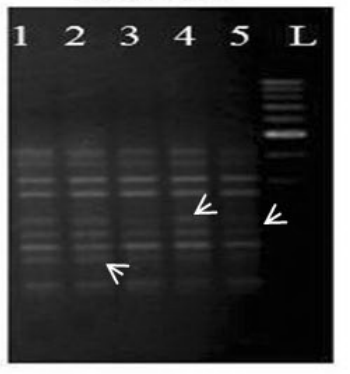

Figure 1. Banding pattern of various primers (primer's name given above the gel photograph) used either individually or on paired arrays with five collections of Cucumis species (lanes 1-5 showing the collection according to Table 1) and arrow head showing the presence of novel/unique bands, which were not amplified by the singleprimer-based RAPD method. 
Table 3. Two RAPD primer combinations used to study the extent of polymorphism at an intra-specific level.

\begin{tabular}{|c|c|c|c|c|}
\hline Primer name & Primer sequence $\left(5^{\prime}-3^{\prime}\right)$ & $\begin{array}{l}\text { Total No. } \\
\text { of bands }\end{array}$ & $\begin{array}{c}\text { No. of polymorphic } \\
\text { bands }\end{array}$ & $\begin{array}{c}\% \text { of } \\
\text { polymorphism }\end{array}$ \\
\hline OPBA-14+ OPBA-16 & TCGGGAGTGG + CCACGCATCA & 6 & 3 & 50 \\
\hline OPAA-11 + OPBA-16 & ACCCGACCTG + CCACGCATCA & 9 & 5 & 55.55 \\
\hline OPAA-12 + OPBA-16 & GGACCTCTTG + CCACGCATCA & 5 & 3 & 60 \\
\hline OPAA-14 + OPD-8 & $\mathrm{AACGGGCCAA}+\mathrm{GTGTGCCCCA}$ & 2 & 1 & 50 \\
\hline OPBA-16 + OPC-11 & CCACGCATCA + AAAGCTGCGG & 10 & 0 & 0 \\
\hline OPAA-11 + OPBA-19 & ACCCGACCTG + CCATCCGTTG & 8 & 5 & 62.5 \\
\hline OPAA-12 + OPBA-19 & GGACCTCTTG + CCATCCGTTG & 5 & 2 & 40 \\
\hline OPC-11 + OPBA-19 & AAAGCTGCGG + CCATCCGTTG & 9 & 6 & 66.66 \\
\hline OPAA-12 + OPAA-1 & GGACCTCTTG + AGACGGCTCC & 6 & 1 & 16.66 \\
\hline OPAA-12 + OPAA-2 & GGACCTCTTG + GAGACCAGAC & 9 & 2 & 22.22 \\
\hline OPAA- $12+$ OPAA- 4 & GGACCTCTTG + AGGACTGCTC & 7 & 2 & 28.57 \\
\hline OPBA-13 + OPAA-2 & AGGGCGAATG + GAGACCAGAC & 6 & 3 & 50 \\
\hline OPBA-13 + OPAA-4 & AGGGCGAATG + AGGACTGCTC & 6 & 2 & 33.33 \\
\hline OPAA-1 + OPAA-4 & AGACGGCTCC + AGGACTGCTC & 6 & 0 & 0 \\
\hline OPBA-13 + OPAA-1 & AGGGCGAATG + AGACGGCTCC & 4 & 4 & 100 \\
\hline OPBA-14 + OPAA-14 & TCGGGAGTGG + AACGGGCCAA & 3 & 2 & 66.66 \\
\hline OPBA-14 + OPC-11 & TCGGGAGTGG + AAAGCTGCGG & 4 & 4 & 100 \\
\hline OPBA-14 + OPD-8 & TCGGGAGTGG + GTGTGCCCCA & 7 & 7 & 100 \\
\hline OPAA-11 + OPAA-12 & ACCCGACCTG + GGACCTCTTG & 4 & 1 & 25 \\
\hline OPAA-11 + OPAA-14 & ACCCGACCTG + AACGGGCCAA & 5 & 3 & 60 \\
\hline OPAA-11 + OPD-8 & ACCCGACCTG + GTGTGCCCCA & 7 & 5 & 71.4 \\
\hline OPAA-12 + OPAA-14 & GGACCTCTTG + AACGGGCCAA & 8 & 8 & 100 \\
\hline OPAA-12 + OPC-11 & GGACCTCTTG + AAAGCTGCGG & 8 & 5 & 62.5 \\
\hline OPAA-11 + OPC-11 & ACCCGACCTG + AAAGCTGCGG & 3 & 3 & 100 \\
\hline OPAA- 12 + OPD-8 & GGACCTCTTG + GTGTGCCCCA & 9 & 9 & 100 \\
\hline OPAA-14 + OPC-11 & AACGGGCCAA + AAAGCTGCGG & 8 & 8 & 100 \\
\hline Total & & 164 & 92 & 56.09 \\
\hline
\end{tabular}

Table 4. Comparison of single-primer RAPD with primer-pair RAPD in terms of band profiles and polymorphisms.

\begin{tabular}{lccccc}
\hline Primers & $\begin{array}{c}\text { Total No. } \\
\text { of bands }\end{array}$ & $\begin{array}{c}\text { No. of polymorphic } \\
\text { bands }\end{array}$ & $\begin{array}{c}\text { Average No. } \\
\text { of bands }\end{array}$ & Genetic distance & \% of polymorphism \\
\hline Primer-pair RAPD method & 164 & 92 & 6.30 & $0.58-0.82$ & 56.09 \\
Single-primer RAPD method & 75 & 41 & 6.25 & $0.67-0.85$ & 54.66 \\
Pooled data of the two approaches above & 239 & 133 & 6.27 & $0.62-0.82$ & 55.64 \\
\hline
\end{tabular}

The pattern of clustering analyzed through Jaccard's distance matrices showed significant variation when data obtained from conventional single-primer RAPD and primer-pair RAPD were compared with each other as well as pooled data of both methods, i.e., single- and primer-pair RAPD, either individually or collectively. The range of distance in conventional RAPD was 0.67-0.85 (Figure 2A) and, in contrast, primer-pair RAPD showed a distance range of 0.58-0.82 (Figure 2B). Interestingly, the distance range in the consensus tree, revealed by pooling data from both methods, was 0.62-0.82 (Figure 2C), which can be considered the mean distance range. Significantly, all the trees obtained either in isolation or in combination did separate the cultivated and wild species of Cucumis showing genetic variation both at intra- and inter-specific levels.

\section{Identification of extra polymorphism and novel bands using primer-pair array}

Interestingly, on the basis of an individual profile, fewer bands were generated when RAPD primers were used singly (conventional method) than in paired arrays. Furthermore, band profiles generated by pairs of random primers did not correspond to the sum of those 


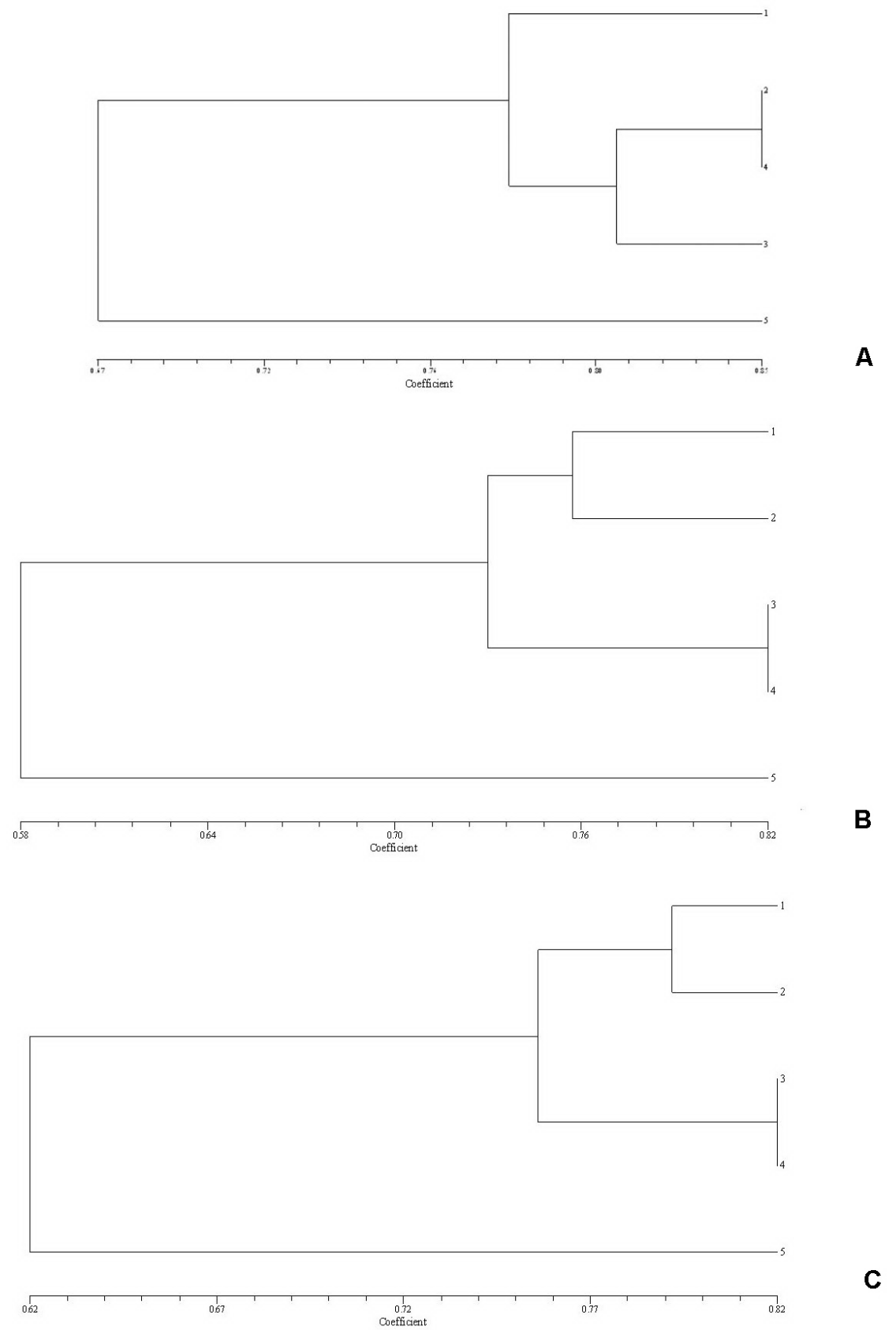

Figure 2. A. Neighbor-joining (NJ) tree generated through conventional single-primer-based RAPD method. B. NJ tree generated through primer-pair arrays. C. NJ tree generated through pooled data of both conventional and primer-pair array methods. 
generated by each primer used alone. The difference was discernable largely in bands that were present in the 'paired' profile but were absent from profiles of either random primer when used separately (novel bands) and in a minority of bands (missed once) that were present in the 'conventional' RAPD profiles but are absent in the paired profiles (lost bands) (Figure 1). Primer-pairs, viz. OPA-11 + OPBA-19, OPBA-16 + OPC-11, OPC-11 + OPBA-19, OPAA-12 + OPAA-4 and OPBA-13 + OPAA-4, showed maximum profile variation and the presence of novel bands with reference to single-primer amplicons for both members of the pair array (Figure 1). On the other hand, some primer-pairs, viz. OPAA-12 + OPA-4 and OPAA-13 + OPAA-4, showed significant loss of amplification products (lost bands) with reference to single-primer amplicons for both members of the pair array (Figure 1). The size of all the novel bands lies between 0.3 and $1.5 \mathrm{~kb}$ in all primer-pair arrays studied presently.

\section{DISCUSSION}

RAPD is a multiplex marker system that conventionally uses single-primer PCR to amplify random DNA fragments (Kumar et al., 2009; Bewal et al., 2009). This is simple, relatively inexpensive, and has been frequently employed to analyze the intra- and inter-specific genetic variation in plants and animals (Yi et al., 2009; Li and Ding, 2010). To overcome the reproducibility problem associated with the conventional RAPD marker, it has been converted into SCAR (Paran and Michelmore, 1993). SCAR markers have been developed for several crops including lettuce (Paran and Michelmore, 1993), common bean (Adam-Blondon et al., 1994), raspberry (Parent and Page, 1995), grape (Reisch et al., 1996), rice (Naqvi and Chattoo, 1996), brassicas (Barret et al., 1998), olive (Hernández et al., 2001), Cynanchum wilfordii, C. auriculatum, and Polygonum multiflorum (Moon et al., 2010).

Very few reports are available on the use of mixtures of the RAPD primers (Sall et al., 2000), which can be used as a new marker linked to various traits governing the genes. Reddy and Soliman (1997) identified the wild and cultivated Hordeum species using the RAPD primer-pair method. Identification of the thickness gene for shuck in walnut ( $\mathrm{Li}$ et al., 2007) and purity testing of $\mathrm{F}_{1}$ hybrid seed in chili pepper (Capsicum annuum; Jang et al., 2004) have been successfully carried out using RAPD and SCAR markers. The same approach has been adopted by Cekic et al. (2001) to demonstrate the potential of ISSR-PCR primer-pair combinations for genetic linkage analysis using the seasonal flowering gene in Fragaria as a model. The primer-pair mixtures have been more frequently used than the conventional RAPD technique in bulk segregant analysis as they generate an extremely large number of markers as was efficiently demonstrated by Sall et al. (2000) using two-primer mixtures. The average number of products increased slightly from the single-primer to the multiple-primer case, but the effect of the competition shows mixtures of more than two primers to be inefficient (Sall et al., 2000).

The present study deals with the utility of the primer-pair reaction in the PCR technique to amplify a relatively large number of bands, which may be distinguished as novel/ unique bands. In the present investigation, it is amply clear that primer-pair RAPD produced a large number (164) of amplification products with a molecular weight range of 0.3 to $2.7 \mathrm{~kb}$, whereas conventional single-primer-based RAPD yielded only 75 bands with a molecular weight range of 0.40 to $2.3 \mathrm{~kb}$. A relatively small amount of primer and other chemicals related to PCR amplification is required in primer-pair RAPD to give better amplification products that encompass a large range of genetic variation. The cluster analysis plotted using Jaccard's 
coefficient showed that both conventional as well as primer-pair method significantly distinguished the cultivated and wild Cucumis species (C. hardwickii) at inter-specific levels. All the accessions belonging to cultivated species (C. sativus) showed significant natural genetic variation at the intra-specific level using both conventional and primer-pair methods. Captivatingly, primer-pair RAPD showed more highly sensitive distance values $(0.67-0.85)$ than conventional RAPD (0.58-0.82).

It is noteworthy that common size fragments in the two primer-pair reactions hybridized with the single-primer reaction products indicate that some fragments amplified by one of the primers were also amplified in the two-primer combination. Therefore, hypothetically, the new bands might have originated from genomic regions that were not amplified in the single-primer reactions.

An overall analysis shows positive discrimination according to the studies of Halldén et al. (1996) and Sall et al. (2000), which describe that competition occurs in all single-primer RAPD reactions as well as multiple-primer reactions. These authors are of the opinion that two-primer reactions yield about 1.3 times more amplicons compared to single-primer reactions. Our study revealed that two-primer RAPD reactions amplified more than two times as many bands as single-primer reactions, which is contradictory to the results of Hu et al. (1995) and Sall et al. (2000). Thus, there is no priori reason to believe that the use of two-primer mixtures in all single-primer-based amplification reactions would generate a higher proportion of erroneous polymorphic bands compared to the use of single-primer mixtures.

By and large, the present investigation demonstrates the usefulness of the primer-pair RAPD method in the generation of new markers for precision genome mapping, which can be developed as sequence-characterized amplified regions linked to various traits and their putative genes. The main advantage of primer-pair RAPD over single-primer-based RAPD is the increase in the total number of reactions and amplification products in the form of novel/ unique bands with a limited number of primers. It is a well-known fact that RAPD primers are relatively low-cost sequences without palindromes as compared to other self-annealing sequence/cycle reactions. However, the major limitation of this approach is that a random primer, which reveals significant polymorphism when used in isolation, may not necessarily produce a higher degree of polymorphism in band patterns when used in pair/combination with another primer. Hence, with the pairing of the primers, the conventional RAPD method can be modified to generate reliable amplicons in large numbers, which can be linked to genegoverning traits, finally leading to partial mapping of the genome.

\section{ACKNOWLEDGMENTS}

E.M. Gatphoh is thankful to the Head of the Department of Biotechnology and Bioinformatics for providing facilities for his dissertation research. Sincere thanks are also extended to Mr. Animos Lamare, technical staff, and to all other members (Ms. Anju, Ms. Merita and Ms. Marlykynti) of the Plant Biotechnology Laboratory, Department of Biotechnology and Bioinformatics for their support and help rendered as and when required.

\section{REFERENCES}

Adam-Blondon AF, Sevignac M, Dron M and Bannerot H (1994). A genetic map of common bean to localize specific resistance genes against anthracnose. Genome 37: 915-924.

Arondel V, Lemieux B, Hwang I, Gibson S, et al. (1992). Map-based cloning of a gene controlling omega-3 fatty acid 
desaturation in Arabidopsis. Science 258: 1353-1355.

Barret P, Delourme R, Foisset N and Renard M (1998). Development of a SCAR (sequence characterised amplified region) marker for molecular tagging of the dwarf Breizh (Bzh) gene in Brassica napus L. Theor. Appl. Genet. 97: 828-833.

Bent AF, Kunkel BN, Dahlbeck D, Brown KL, et al. (1994). RPS2 of Arabidopsis thaliana: a leucine-rich repeat class of plant disease resistance genes. Science 265: 1856-1860.

Bewal S, Sharma SK, Parida A, Shivam S, et al. (2009). Utilization of RAPD marker to analyse natural genetic variation in Calligonum polygonoides L. - a key stone species of Thar desert. Int. J. Integ. Biol. 5: 148-151.

Caetano-Anolles G, Bassam BJ and Gresshoff PM (1991). DNA amplification fingerprinting using very short arbitrary oligonucleotide primers. Biotechnology 9: 553-557.

Cekic C, Battey NH and Wilkinson MJ (2001). The potential of ISSR-PCR primer-pair combinations for genetic linkage analysis using the seasonal flowering locus in Fragaria as a model. Theor. Appl. Genet. 103: 540-546.

Cushwa WT and Medrano JF (1996). Applications of the random amplified polymorphic DNA (RAPD) assay for genetic analysis of livestock species. Anim. Biotechnol. 7: 11-31.

Halldén C, Hansen M, Nilsson NO, Hjerdin A, et al. (1996). Competition as a source of errors in RAPD analysis. Theor. Appl. Genet. 93: 1185-1192.

Hernández P, de la Rosa R, Rallo L, Martín A, et al. (2001). First evidence of a retrotransposon-like element in olive (Olea europaea): implications in plant variety identification by SCAR-marker development. Theor. Appl. Genet. 102: 1082-1087.

Hu J, van Eysden J and Quiros CF (1995). Generation of DNA-based markers in specific genome regions by two-primer RAPD reactions. PCR Methods Appl. 4: 346-351.

Huang MC, Horng YM, Huang HL, Sin YL, et al. (2003). RAPD fingerprinting for the species identification of animals. Asian Austral. J. Anim. 16: 1406-1410.

Hunt GJ and Page RE Jr (1995). Linkage map of the honey bee, Apis mellifera, based on RAPD markers. Genetics 139: 1371-1382.

Innis MA, Gelfand DH and Sninsky JJ (1990). PCR Protocols: A Guide to Methods and Applications. Academic Press, New York.

Jaccard P (1901). Étude comparative de la distribution florale dans une portion des Alpes et des Jura. Bull. Soc. Vaudoise Sci. Nat. 37: 547-579.

Jang I, Moon JH, Yoon JB, Yoo JH, et al. (2004). Application of RAPD and SCAR markers for purity testing of F1 hybrid seed in chili pepper (Capsicum annuum). Mol. Cells 18: 295-299.

Kumar RV, Tripathi YK, Shukla P, Ahlawat SP, et al. (2009). Genetic diversity and relationships among germplasm of Jatropha curcas L. revealed by RAPDs. Trees - Struct. Funct. 23: 1075-1079.

Li Y and Ding WL (2010). Genetic Diversity assessment of Trollius accessions in China by RAPD markers. Biochem. Genet. 48: 34-43.

Li Z, Lanying Z and Qianwen X (2007). Identification of RAPD markers linked to thickness gene of shuck in walnut. $A d v$. Biol. Res. 1: 137-140.

Moon BC, Choo BK, Cheon MS, Yoon T, et al. (2010). Rapid molecular authentication of three medicinal plant species, Cynanchum wilfordii, Cynanchum auriculatum, and Polygonum multiflorum (Fallopia multiflorum), by the development of RAPD-derived SCAR markers and multiplex-PCR. Plant Biotechnol. Rep. 4: 1-7.

Murray MG and Thompson WF (1980). Rapid isolation of high molecular weight plant DNA. Nucleic Acids Res. 8: 4321-4325.

Naqvi NI and Chattoo BB (1996). Development of a sequence characterized amplified region (SCAR) based indirect selection method for a dominant blast-resistance gene in rice. Genome 39: 26-30.

Paran I and Michelmore RW (1993). Development of reliable PCR-based markers linked to downy mildew resistance genes in lettuce. Theor. Appl. Genet. 85: 985-993.

Parent JG and Page D (1995). Evaluation of SCAR markers to identify raspberry cultivars. Hort. Sci. 30: 856.

Reddy PV and Soliman KM (1997). Identification of wild and cultivated Hordeum species using two-primer RAPD fragments. Biol. Plant. 39: 543-552.

Reisch BI, Weeden NF, Lodhi MA and Ye G (1996). Linkage Map Construction in Two Hybrid Grapevine (Vitis sp.) Populations. In: Plant Genome IV: Proceedings of the Fourth International Conference on the Status of Plant Genome Research, College Park, 26.

Sall T, Lind-Hallden C and Hallden C (2000). Primer mixtures in RAPD analysis. Hereditas 132: 203-208.

Sharma SK, Rawat D, Kumar S, Kumar A, et al. (2010). Single primer amplification reaction (SPAR) reveals intra-specific natural variation in Prosopis cineraria (L.) Druce. Trees - Struct. Funct. 24: 855-864.

Welsh J and McClelland M (1990). Fingerprinting genomes using PCR with arbitrary primers. Nucleic Acids Res. 18: 7213-7218.

Williams JG, Kubelik AR, Livak KJ, Rafalski JA, et al. (1990). DNA polymorphisms amplified by arbitrary primers are 
useful as genetic markers. Nucleic Acids Res. 18: 6531-6535.

Williams JG, Reiter RS, Young RM and Scolnik PA (1993). Genetic mapping of mutations using phenotypic pools and mapped RAPD markers. Nucleic Acids Res. 21: 2697-2702.

Yi SS, Akashi Y, Tanaka K, Cho TT, et al. (2009). Molecular analysis of genetic diversity in melon landraces (Cucumis melo L.) from Myanmar and their relationship with melon germplasm from East and South Asia. Genet. Resour. Crop Evol. 56: 1149-1161. 